\title{
Wide-WANET Architecture for Extension of Internet Facilities in Remote Regions
}

\author{
Pradeep Kumar Gaur, Anupma Marwaha
}

\begin{abstract}
A definitive research of intrigue nowadays is in the field of internetworking identified with overseeing and keeping up of correspondence between nodes of various network standards inside an ad-hoc domain. The communication between heterogeneous nodes is established by the computation of the instantaneously selected metric values i.e. hop count and routing queue length of individual gateways candidates. A wide-WANET structure is proposed conforming to four different network standards and simulated using enhanced AODV and IPv6 addressing format. It has been proved through simulations that the parameters in consideration has shown improved better performance by implementing Multiple-metrics Gateway Selection Criterion whereby facilitating extension of Internet facilities by cohering nodes of varying network standards.
\end{abstract}

Index Terms: Hop Count; Infrastructure less; Multiple-metrics Gateway Selection Criterion; Routing Queue Length; wideWANET;

\section{INTRODUCTION}

There has been a curious interest amongst novice engineers to link electronic devices with the Internet and control their working at different instances of time at will. But the source of Internet may or may not be infrastructure full indicating that some areas in a locality could be devoid of DSL and ADSL Links as a source of Internet.

This is where arises the need for integrating devices configured in various network standards viz. ZigBee, UMTS etc. [1] forming a network structure nomenclated as wide WANET. The given devices are identified by assigning IPv6 addresses using auto-configuration algorithm and getcoalesced to form a virtual large scale wireless ad-hoc network.

A network architecture as shown in Figure 1 is proposed for simulation and to implement the suggested scenario. The ever increasing demand of IP Addresses is taken care of by the IPv6 protocol and the across the network connectivity is activated by discovery of Wireless gateway with dual stack provision [2, 3, 4]. Extended form of MIP i.e. Mobile IP is used to connect and manage mobile hosts moving into foreign networks from the parent ad-hoc network [5]. It has been the need of the hour to combine the functioning of next generation devices and systems to maintain backward compatibility. The authors exclaimed the importance of ad-hoc networks as a solution to increase the radio coverage for providing multimedia services and topology control in wireless domain $[6,7,8]$.

Bluetooth based low energy radios due to their increasing ubiquitous presence are used to connect IoT devices to Internet.

Revised Manuscript Received on June 15, 2019.

Pradeep Kumar Gaur, Electronics and Communication Engineering,

Chandigarh Engineering College, Landran, Mohali, Punjab, India.

Anupma Marwaha, Electronics and Communication Engineering, SLIET, Longowal, Sangrur, Punjab, India
There are still many challenges left to be explored in their implementation \& deployment at application layer $[9,10]$. In [11], a low power and low cost gateway has been designed and implemented as a custom hardware platform acting as a gateway that has sufficient resources to support a multitasking operating system such as Linux or Windows Embedded CE.

Authors in [12] integrated the low-VHF radio system and a conventional ZigBee System on a small sized robotic platform for self-sustained experimentation using highly miniaturized efficient antenna.

From hardware to software implementation of network gateways emancipated so far, the generation and development of an ad-hoc models which can overcome compatibility issues has been taken into consideration, hence in the current research work, a wide-WANET architecture is proposed and implemented such that the Internet facilities can happen to serve the under-privileged people dwelling in far flung regions.

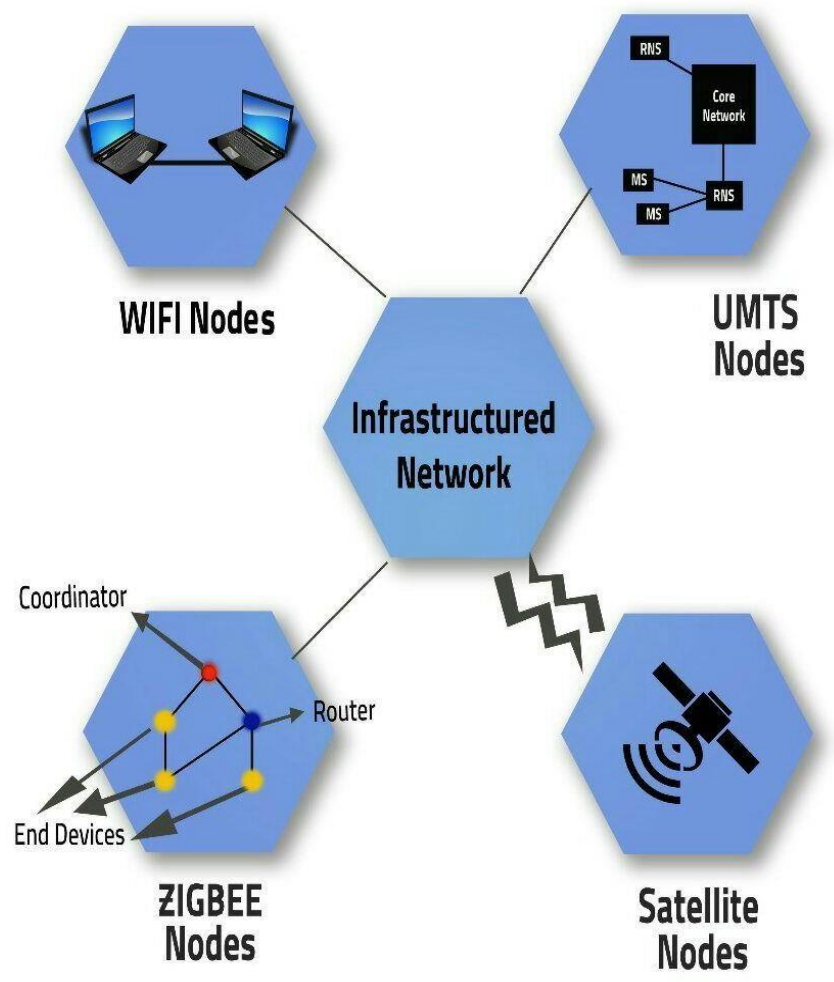

Fig. 1: Proposed Network Architecture: A wide WANET [1] 


\section{METHODOLOGY}

Heterogeneous networks behave differently as per their defined logical structures hence following procedural stages has been framed to ease the discovery of gateway nodes in given networks at different instances of time and scenarios. Four network scenario [1] are designed in NS2 forming a wide WANET to transact a constant byte packet from and to the fixed Internet source. The nodes of heterogeneous networks have been randomly placed in ad-hoc environment and the gateway is independently chosen for communicating packets with the fixed network and mobile node e.g. end devices in ZigBee, Mobile Stations (MS) in UMTS systems and the satellite nodes. Two level hierarchy is maintained since nodes of one network are supposed to acknowledge the gateway of the other network meaning thereby only the network address is required instead of the host address. The IPv6 auto-configuration protocol is used to allocate 128-bit addresses to the nodes to automatically assign IP addresses to the nodes being stimulated in Ad-hoc environment and 64-bit prefix used is of non-mac character and is a random number to avoid duplicity. The enhanced version of AODV with auto-address assignment capability is used at local level in individual sub networks to sustain intra communication and then at the global level i.e. the whole wide WANET for maintaining inter communication through the assignment of gateway function. The most critical part of implementation is discovery and selection of gateway[13]. The probable gateways are selected using Multiple-metrics Gateway Selection Criterion (MGSC) using Simple Additive Weighing Scheme. Multiple metrics. All the nodes in different networks implement the selection criterion by assigning weights to multiple metrics independently and automatically. The metrics are considered to have varying ranges and unique value units specified in the unused fields of the packet. Therefore the metrics have to be scaled into different non-dimensional values. An improved version of AODV with HELLO packets is used for gateway discovery. The life time of HELLO packets is 1 and hence all the nodes in a sub network may not access it in one go. These packets are a derivate of RREP (route reply) packets, a number of fields are perceived to be free. Initially a modified HELLO Packet is sent by a gateway with a set flag named as IFlag mentioning the gateway address to depict its origination. Such packets are nomenclated as HELLO_I packets being routed to every node within range to enable the setting of the gateway in their routing table as a next hop entry for communication with the nodes in infrastructure full network. There are chances that all the available active nodes may not receive the HELLO_I packet within its HELLO interval, affected node may broadcast a simple HELLO packet instead of a HELLO_I packet to ascertain the nearest gateway. To evaluate the consistency of the new MGSC scheme working in consonance with AODV protocol, following two parameters as defined.

$$
\begin{aligned}
& \text { Average Packet Drop }=\frac{\text { Number of packet drops }}{\text { Number of packet arrivals }} \\
& \text { Throughput }=\frac{\text { Number of bytes received } \times 8}{\text { Simulation time } \times 1000} \mathrm{Kbps}
\end{aligned}
$$

\section{RESULTS and DISCUSSION}

The NS2 tool is used to simulate heterogeneous nodes using enhanced AODV updated with MGSC scheme for gateway selection. The constant bit rate traffic is used with a packet size of 512 Bytes with nodes of omni- directional antenna type for creating a virtual wireless channel working in ad-hoc fashion as depicted in Table I.

\begin{tabular}{|c|c|}
\hline Parameters & Values \\
\hline Number of Nodes & $25,50,75,100,125$ \\
\hline Addressing Type & IPv6, Hierarchical \\
\hline IPv6 Simulation Time & $\begin{array}{l}\text { 300s (for showing } \\
\text { scalability) }\end{array}$ \\
\hline Traffic type/ Packet Size & CBR/ 512 Bytes \\
\hline Simulation Area & $1000 \mathrm{~m} * 1000 \mathrm{~m}$ \\
\hline Routing Protocol & Enhanced AODV \\
\hline MAC Protocol & $\begin{array}{l}\text { 802.11, Satellite, } \\
\text { 802.15.4, UMTS }\end{array}$ \\
\hline Interface Queue Type & Queue/DropTail \\
\hline Interface Queue Length & 100 \\
\hline Antenna Type & $\begin{array}{l}\text { Omni-directional } \\
\text { Antenna }\end{array}$ \\
\hline Propagation Model & $\begin{array}{l}2 \text { Ray Ground } \\
\text { propagation }\end{array}$ \\
\hline Physical Interface Type & Phy/ WirelessPhy \\
\hline Channel & Wireless \\
\hline
\end{tabular}

Table I: Simulation Parameters

Normal AODV [14] uses the Hop Count metric to identify the gateway, the results of which are compared with enhanced AODV implemented with additional metric called routing queue length. 


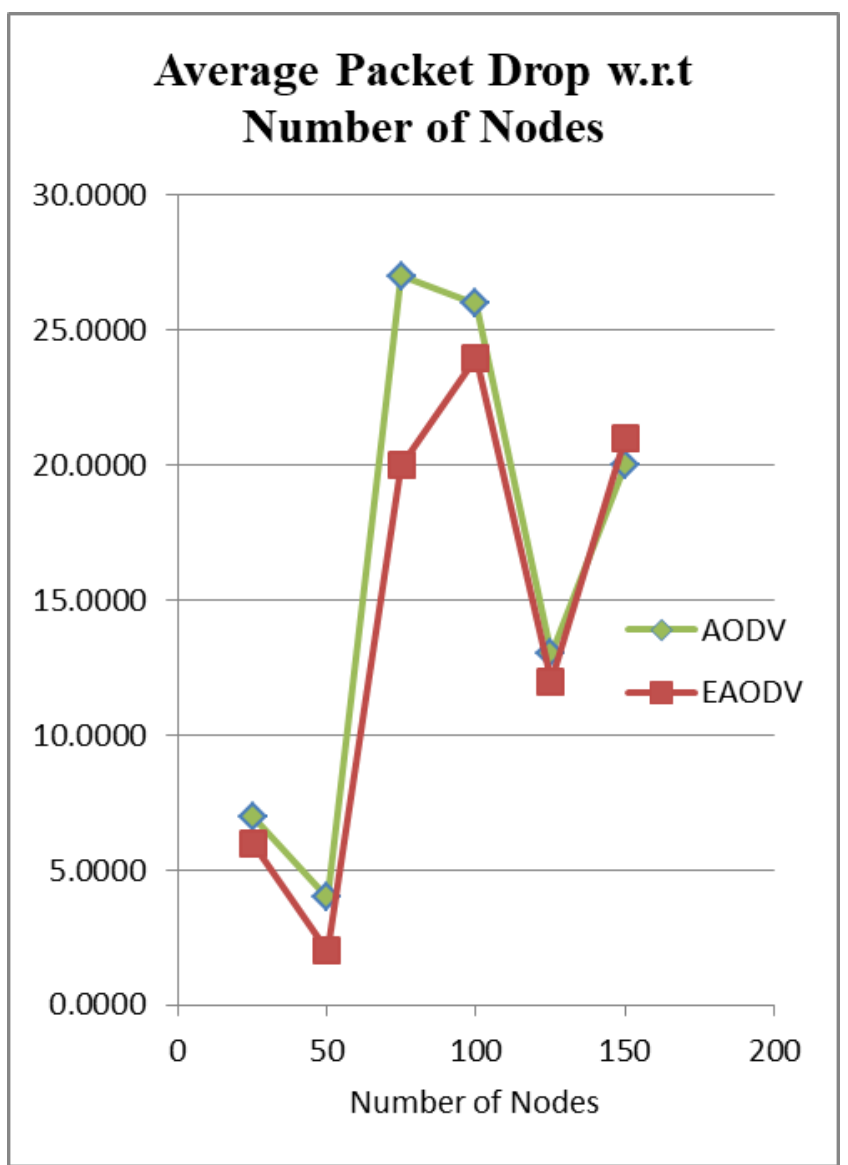

Fig. 2: Average packet drop w.r.t number of nodes

Figure 2 shows that the average packet drop is comparable for MGSC scheme implemented as part of along with enhanced AODV (EAODV) and normal AODV but the average packet drop increases when the scenario is implemented for over 100 nodes as the simulation time is constant for 300 seconds. The scenario area is even fixed so the simulation for increasing number of nodes shows more and more congestion resulting in more packet drop.

Throughput in MGSC scheme implementation produces better throughput as the gateway is selected keeping into account the routing queue length which includes the advertisement overheads as well as shown in Figure 3. But the increasing number of nodes in overall scenario shows increasing variations due to congestion and random movement of nodes. So overhead is understood to increase in multi-metric based simulation implemented with EAODV in comparison to simulations with normal AODV and the throughput is marginalized with the increasing number of nodes.

\section{CONCLUSION}

A framework of wide-WANET is designed in NS2 wherein the gateways are selected as per the policy framed acronym as MGSC Scheme in the current work. The efficacy of the implemented MGSC Scheme in cohesion with enhanced AODV for selection of gateway nodes is proved upon simulation of the well-defined virtual wide-WANET architecture.

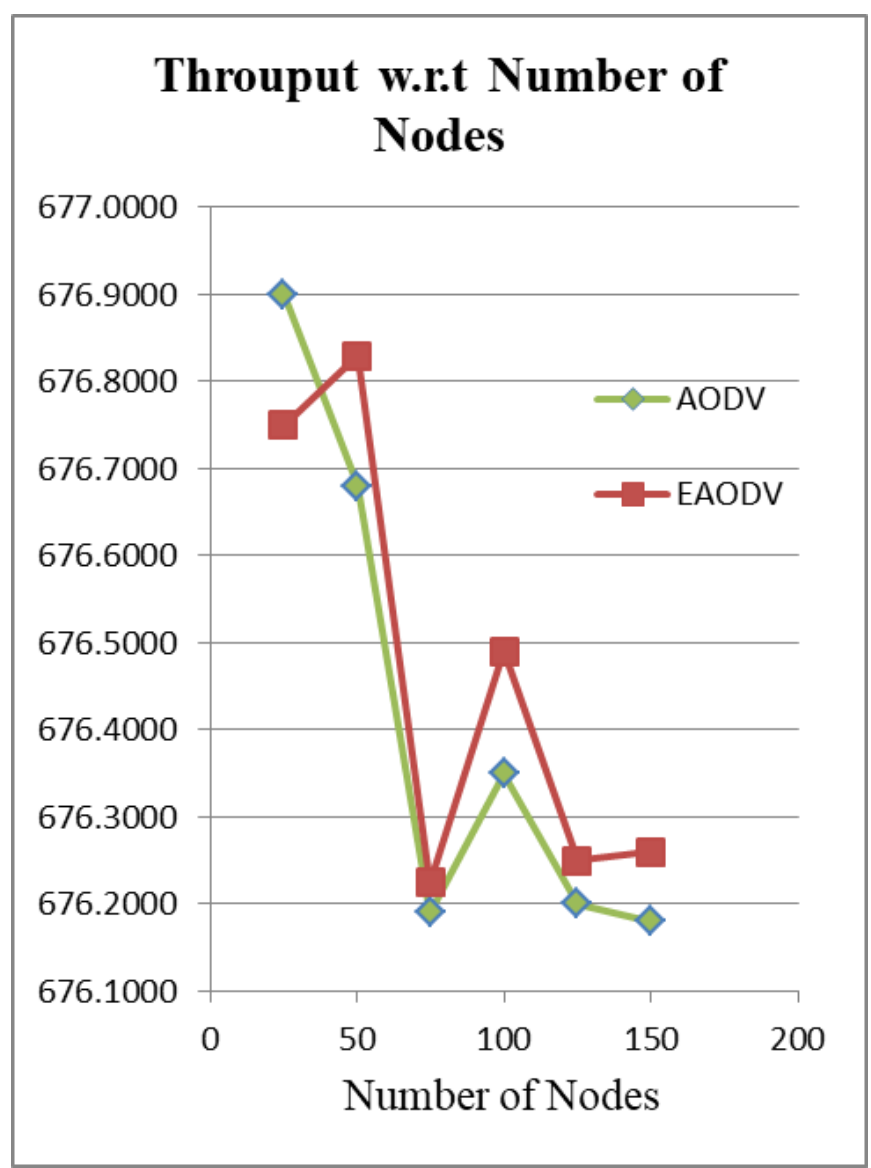

Fig. 3: Throughput w.r.t. number of nodes

The research is still continuing to make the MGSC scheme more robust, adequate and productive by fusing the third parameter namely 'remaining node energy' within the suggested framed policy to facilitate the ever increasing Internet demands in remote locations meanwhile developing enhanced AODV protocol.

\section{REFERENCES}

1. Gaur PK, Marwaha A "Scalability Analysis using Auto-configuration process for coalescing of heterogeneous Wireless ad-hoc networks" International Journal of Applied Engineering Research, Research India Publications, 12(14), 2007, pp. 4427-4432.

2. Bayer N, Xu B, Hischke S, "An Architecture for connecting Ad hoc Networks with the IPv6 Backbone (6Bone) using a Wireless Gateway", European Wireless, vol. 100, 2004.

3. Khan KUR, Reddy AV, Zaman RU, Kumar M, "An effective gateway discovery mechanism in an integrated internet-MANET (IIM)" Int Conf Adv Comput Eng, 2010, pp. 24-28.

4. Ratanchandani P, Kravets R, "A Hybrid Approach to Internet Connectivity for Mobile Ad Hoc Networks", Wirel Commun Netw, vol. 3, 2003, pp.1522-1527.

5. Ahlund C, Zaslavsky A, "Integration of ad hoc network and IP network capabilities for mobile hosts", 10th Int Conf Telecommun ICT, vol. 1, 2003, pp. 482-489. doi: 10.1109/ICTEL.2003.1191288

6. Armuelles I, Robles T, Siebert M, "On Ad Hoc Networks in the 4G Integration Process", Third Annual Mediterranean Ad Hoc Networking Workshop,

2004. 
7. Bader F, Pinart C, Christophi C, "User-centric analysis of perceived QoS in 4G IP mobile/wireless networks" IEEE Int Symp Pers Indoor Mob Radio Commun PIMRC, vol. 3, 2003, pp. 2047-2053.

8. Djouama A, Mokdad L, Abdennebi M, Tohmé S, "Topology control for enhanced QoS on infrastructure-less heterogeneous radio networks" Proc Conf Local Comput Networks, LCN, 2009, pp. 414-419.

9. Zachariah T, Klugman N, Campbell B, "The Internet of Things Has a Gateway Problem", Proc 16th Int Work Mob Comput Syst Appl HotMobile'15, 2015, pp. 27-32.

10. Glória A, Cercas F, Souto N, "Design and implementation of an IoT gateway to create smart environments", Procedia Comput Sci, vol. 109, 2017, pp. 568-575.

11. Villiers G De, Byl A Van der, Wilkinson RH, "Developing a WSN internet gateway for an African context", Int J Sens Networks, vol. 20(1), 2016.

12. Choi J, Dagefu FT, Sadler BM, Sarabandi K, "Low-Power Low-VHF AdHoc Networking in Complex Environments", IEEE Access, vol. 5, 2017, pp. 24120-24127.

13. El-Moshrify H, Mangoud MA, Rizk M, "Gateway discovery in Ad hoc On-Demand Distance Vector (AODV) routing for internet connectivity", Natl Radio Sci ConfNRSC, Proc, vol 2, 2007, pp. 1-8.

14. Perkins, C. E., Royer, E. M. \& Das, S., "Ad hoc on demand distance vector (AODV) routing for IP version 6" Internet Draft, 2000

\section{AUTHORS PROFILE}

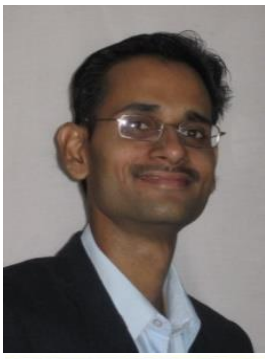

Dr. Pradeep K Gaur is Associate professor in Electronics and Communication Engineering Department at Chandigarh Engineering College, Landran, Mohali. He obtained his B.Tech in ECE from AIET Faridkot and M.Tech Degree in the same field from GNDEC, Ludhiana in 2001 and 2009 respectively. Currently He is pursuing his $\mathrm{Ph} . \mathrm{D}$ from SLIET Longowal in the domain of Wireless Ad-hoc Networks. He is researching in the area of protocol development for integration of networks using efficient discovery mechanisms.

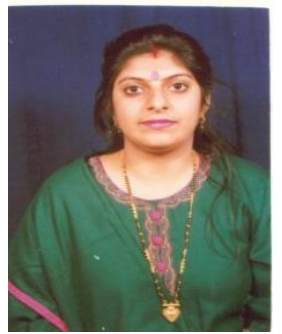

Dr. (Mrs.) Anupma Marwaha is professor and head in Electronics and Communication Communication Engineering Department at SLIET Longowal, Distt. Sangrur, Punjab a deemed university under Ministry of HRD. She obtained her B. E in Electronics \& Comm. Engg. From Punjab Engineering College, Chandigarh and M.Tech Degree in the same field in 1990 and 1992 respectively. She completed her Ph. D. Degree in Electronics from GND University, Amritsar in the year 2003 and specialization in the 'Design and Field Analysis of Electromagnetic Devices by Finite Element Method with applications to Communication Engg., Microwave and Antennas'. She is a Life Member of ISTE, New Delhi and Member of Institution of Engineers, India. She has more than 80 publications to her credit in refereed International and National Journals. .Her continuing research work primarily focuses on developing bioelectromagnetic therapeutics at microwave frequencies and strategically development of hybrid protocols for cohesion of IPv6 Addressing in Wireless Ad-hoc Networks. 